\title{
Shear-Flow-Induced Unfolding of Polymeric Globules
}

\author{
A. Alexander-Katz, ${ }^{1}$ M. F. Schneider, ${ }^{2}$ S. W. Schneider, ${ }^{3}$ A. Wixforth, ${ }^{2}$ and R. R. Netz ${ }^{1}$ \\ ${ }^{1}$ Physics Department, Technical University Munich, 85748 Garching, Germany \\ ${ }^{2}$ University of Augsburg, Experimental Physics I, 86159 Augsburg, Germany \\ ${ }^{3}$ Department of Dermatology, University of Münster, 48149 Münster, Germany
}

(Received 23 March 2006; published 25 September 2006)

\begin{abstract}
The behavior of a single collapsed polymer under shear flow is examined using hydrodynamic simulations and scaling arguments. Below a threshold shear rate $\dot{\gamma}^{*}$, the chain remains collapsed and only deforms slightly, while above $\dot{\gamma}^{*}$ the globule exhibits unfolding/refolding cycles. Hydrodynamics are crucial: In the free draining case, $\dot{\gamma}^{*}$ scales with the globule radius $R$ as $\dot{\gamma}^{*} \sim R^{-1}$, while in the presence of hydrodynamic interactions $\dot{\gamma}^{*} \sim R$. Experiments on the globular von Willebrand protein confirm the presence of an unfolding transition at a well-defined critical shear rate.
\end{abstract}

DOI: 10.1103/PhysRevLett.97.138101

PACS numbers: $87.15 . \mathrm{He}, 82.35 . \mathrm{Lr}$

The dynamics of polymers subject to shear flow has been intensely studied due to its fundamental and practical relevance. Consideration was given to single polymers [1-3] as well as to polymer melts [4]. One conclusion was that polymers under good solvent conditions in simple shear flow show pronounced conformational fluctuations, exhibiting periodic elongation, relaxation, and tumbling, even at relatively small shear rates of the order of the inverse polymer relaxation time [2,5]. It was shown that the mean extension varies smoothly with shear rate, and, thus, no well-defined deformation transition exists $[1,2]$. For polymers under bad solvent conditions, on the other hand, grafted chains were predicted to exhibit a globulestretch transition at a critical shear rate [6]. However, the behavior of free (i.e., nongrafted) globular polymers subject to shear flow has received little attention. This is surprising in light of the direct relation to protein stability under shear flow, a subject of immediate physiological and technological relevance.

Our motivation comes from recent studies on the von Willebrand factor (vWF), a large multimeric glycoprotein that is found in blood plasma and connective tissue of blood vessels. The vWF is assembled via covalent disulfide bonds from hundreds of monomeric units, each consisting of 2813 amino acids, and is one of the largest soluble proteins known [7]. The vWF plays a central yet not fully understood role in the process of blood clotting; its activity seems to be regulated by hydrodynamic shear, which in small arterioles is of the order of $\dot{\gamma} \simeq 1000 \mathrm{~s}^{-1}$ and reaches up to $\dot{\gamma} \simeq 10000 \mathrm{~s}^{-1}$ in partly clogged coronary arterioles [8]. Single-molecule studies of surfaceadsorbed vWF revealed that fluid shear rates of about $3000 \mathrm{~s}^{-1}$ are sufficient to unfold vWF into thin fibers [9], suggestive of a close function-conformation relationship in vWF physiological activities. Video-microscopy data for the stretching of fluorescently labeled vWF in a microflow chamber, which are compared with our simulation studies in this Letter, demonstrated recently that shear-induced unfolding of vWF also occurs in bulk at comparable shear rates of about $5000 \mathrm{~s}^{-1}$ and thus requires no surface binding [10]. In this Letter, we study the mechanism of shearinduced globule unfolding and, in particular, work out the dependence of the critical shear rate on the globule size and cohesive strength using scaling and simulation, in both the presence and the absence of hydrodynamic effects. The mechanism we propose is different from the breakup of fluid spheres in shear [11] and involves the coupling between hydrodynamic drag and globule fluctuations in the form of thermally induced polymeric protrusions that transiently form at the surface of the rotating globule. For collapsed polymers, the unfolding transition appears as a rather sharp increase of the mean polymer extension at a well-defined critical shear rate, in qualitative agreement with experimental results for the vWF.

In our model, the polymer is made of $N$ beads of radius $a$ interacting through a potential $U$. The dynamics of the $i$ th bead position $\mathbf{r}_{i}$ is given by the Langevin equation

$$
\frac{\partial}{\partial t} \mathbf{r}_{i}=\mathbf{v}\left(\mathbf{r}_{i}\right)-\sum_{j} \boldsymbol{\mu}_{i j} \cdot \nabla_{\mathbf{r}_{j}} U(t)+\boldsymbol{\xi}_{i}(t)
$$

where $\mathbf{v}(\mathbf{r})$ is the undisturbed solvent-flow profile, and $\boldsymbol{\xi}_{i}$ is a vector random force that satisfies $\left\langle\boldsymbol{\xi}_{i}(t) \boldsymbol{\xi}_{j}\left(t^{\prime}\right)\right\rangle=$ $2 k_{B} T \boldsymbol{\mu}_{i j} \delta\left(t-t^{\prime}\right)$. For simple shear flow $\mathbf{v}(\mathbf{r})=\dot{\gamma} z \hat{\mathbf{x}}$, where $\dot{\gamma}$ is the shear rate, $z$ the height, and $\hat{\mathbf{x}}$ the unit vector parallel to the $x$ axis. Hydrodynamic interactions are captured through the mobility matrix $\boldsymbol{\mu}_{i j}$. We consider two scenarios: the free draining (FD) model that neglects hydrodynamic interactions between monomers with a mobility matrix given by $\boldsymbol{\mu}_{i j}=\mu_{0} \delta_{i j} \mathbf{1}$, where $\mu_{0}$ is the mobility of a sphere of radius $a$, i.e., $\mu_{0}=1 / 6 \pi \eta a$ ( $\eta$ denotes the viscosity of the fluid). The other case is the hydrodynamic interacting (HI) model where the mobility matrix is given by the Rotne-Prager tensor, which correctly accounts for long range hydrodynamic effects and solvent-flow stagnation inside the globule [3,12]. Comparing results for the two models allows one to access the importance of hydrodynamics. The potential energy $U=U_{s}+U_{\mathrm{LJ}}$ accounts 


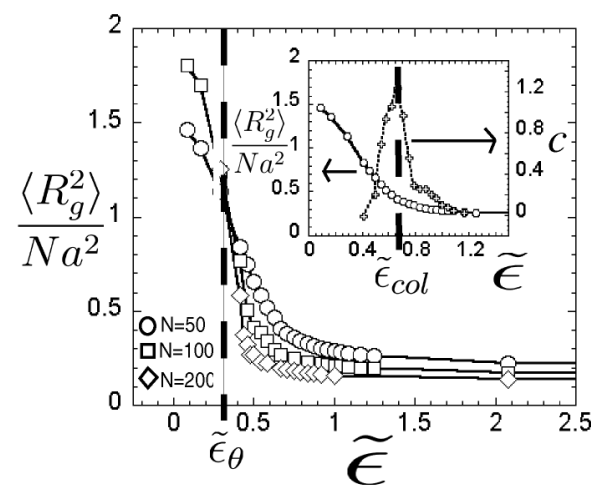

FIG. 1. Rescaled radius of gyration $R_{g}^{2} / N a^{2}$ as a function of the attraction strength $\tilde{\epsilon}$ in the absence of shear. The inset shows data for $N=50$ together with the curvature $c=$ $\partial^{2}\left\langle R_{g}^{2} / N a^{2}\right\rangle / \partial \tilde{\epsilon}^{2}$. The theta point and the maximum curvature point are denoted by $\tilde{\epsilon}_{\theta}$ and $\tilde{\epsilon}_{\text {col }}$, respectively.

for the connectivity of the chain, $U_{s}=$ $\frac{\kappa}{2} k_{B} T \sum_{i=1}^{N-1}\left(r_{i+1, i}-2 a\right)^{2}$, where $r_{i+1, i}$ is the distance between adjacent beads along the chain, and the spring constant is taken to be $\kappa=200 / a^{2}$, which limits stretching of the chain. The second term is a Lennard-Jones potential $U_{\mathrm{LJ}}=\tilde{\epsilon} k_{B} T \sum_{i j}\left[\left(2 a / r_{i, j}\right)^{12}-2\left(2 a / r_{i, j}\right)^{6}\right]$, where $\tilde{\boldsymbol{\epsilon}}$ determines the depth of the potential (in units of $k_{B} T$ ) and $r_{i, j}$ is the distance between the $i$ th and the $j$ th bead. We discretize Eq. (1) with a time step ranging from $10^{-4} \tau$ to $10^{-6} \tau$, where $\tau$ is the characteristic monomer diffusion time $\tau=$ $a^{2} / \mu_{0} k_{B} T$. Averages are taken over a total number of Langevin steps of at least $2 \times 10^{7}$, with the first $10^{6}$ simulation steps typically discarded for equilibration.

In the absence of shear, we define the collapse point $\tilde{\boldsymbol{\epsilon}}_{\mathrm{col}}$ as the maximum in the curvature of the radius of gyration $c=\partial^{2}\left\langle R_{g}^{2} / N a^{2}\right\rangle / \partial \tilde{\boldsymbol{\epsilon}}^{2}$ (Fig. 1). For a polymer with $N=$ 50 , we find $\tilde{\epsilon}_{\text {col }} \sim 0.66$, in close agreement with previous simulation studies [13]. In the limit $N \rightarrow \infty$, the collapse point $\tilde{\boldsymbol{\epsilon}}_{\mathrm{col}}$ approaches the $\theta$ temperature, located at $\tilde{\epsilon}_{\text {col }}^{N \rightarrow \infty}=\tilde{\epsilon}_{\theta} \approx 0.314$ [14], at which the polymer is ideal. For a finite chain length, $\tilde{\epsilon}_{\text {col }}$ gives a heuristic but robust measure of the threshold of chain compactification.

At the top of Fig. 2, we show snapshots of a collapsed polymer $(\tilde{\boldsymbol{\epsilon}}=2.08)$ at a shear rate $\dot{\gamma} \tau=1.2$ slightly above the unfolding threshold. The upper row displays the formation of a protrusion (encircled beads) that eventually leads to a stretch-fold event. Below, we present time sequences of the extension $R_{s}$ (defined as the projected polymer length along the flow direction; see snapshots) for three different shear rates. $R_{s}$ is measured directly in experiments with fluorescently labeled polymers [2,5]. One discerns two dynamical regimes: For low shear rate $\dot{\gamma} \tau=0.5$ (black trace in the first sequence), the chain remains collapsed, in contrast to an uncollapsed polymer $\tilde{\boldsymbol{\epsilon}}=0.416$ (light gray curve in the upper sequence) where strong length fluctuations are observed. As the shear rate $\dot{\gamma}$ is increased to $\dot{\gamma} \tau=1.2$, the chain starts exhibiting pronounced and repeated elongation and folding events, as
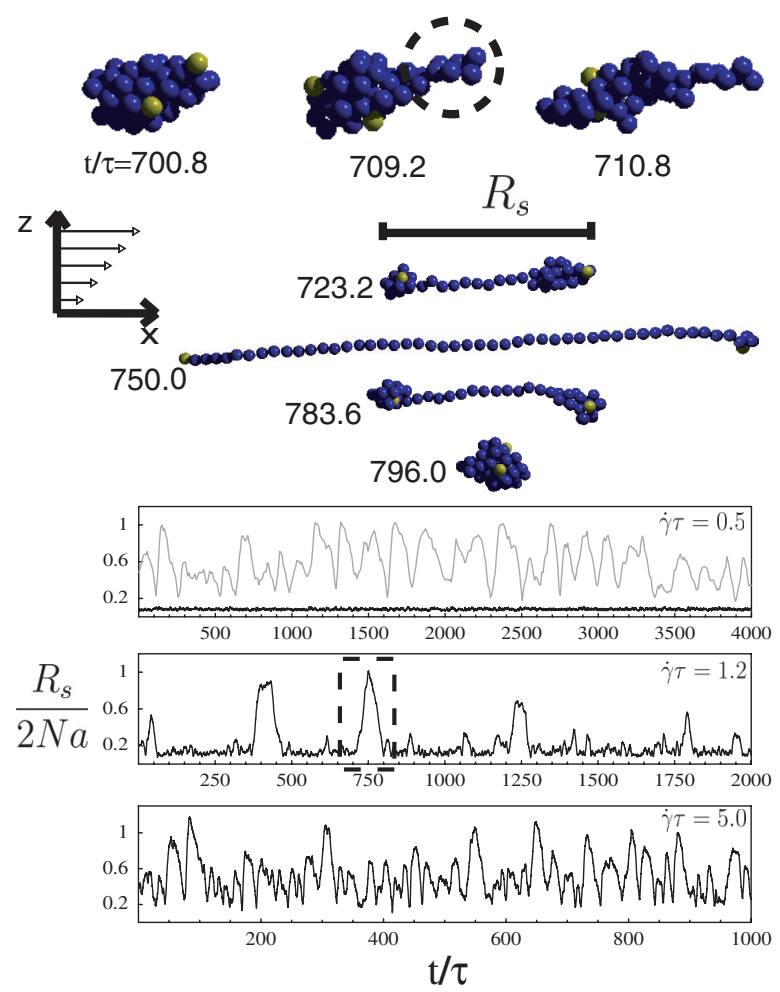

FIG. 2 (color online). Top: Snapshots of a polymer with $N=$ 50 and $\tilde{\boldsymbol{\epsilon}}=2.08$ undergoing stretch-folding events at a dimensionless shear rate of $\dot{\gamma} \tau=1.2$. The upper row shows the formation of a protrusion (encircled beads), followed by a stretch-fold event displayed below. Bottom: Typical extension sequences as a function of time for the FD case for $\tilde{\epsilon}=2.08$ and shear rates $\dot{\gamma} \tau=0.5,1.2$, and 5. For comparison, we also show data for $\dot{\gamma} \tau=0.5$ for an uncollapsed polymer with $\tilde{\epsilon}=0.416$ (light gray curve). The dashed rectangle denotes the stretching event from which the snapshots were taken.

shown in the middle panel and the snapshots. Further increase of the shear rate to $\dot{\gamma} \tau=5.0$ leads to a higher frequency of stretching events.

In Fig. 3, we show the mean extension $\left\langle R_{s}\right\rangle$ for a chain with $N=50$ as a function of the dimensionless shear rate $\dot{\gamma} \tau$ for different values of the attraction strength $\tilde{\boldsymbol{\epsilon}}$. FD and HI simulations are distinguished by open and solid symbols, respectively. As seen, a collapsed chain undergoes a transition to an extended state beyond a "critical" shear rate $\dot{\gamma}^{*}$ in both models, and this transition sharpens as the cohesive strength increases (for comparison, the data for an uncollapsed polymer with $\tilde{\varepsilon}=0.41$, open circles, exhibits merely a smooth unfolding crossover). The transition point $\dot{\gamma}^{*}$ is defined by the maximum of the variance of the squared chain stretching $m=\left(\left\langle R_{s}^{4}\right\rangle-\left\langle R_{s}^{2}\right\rangle^{2}\right) /\left\langle R_{s}^{2}\right\rangle^{2}$. For the particular case $N=50, \tilde{\boldsymbol{\epsilon}}=1.25, m$ is denoted by crosses and exhibits a sharp peak at $\dot{\gamma}^{*} \tau \approx 0.1$. Squares in Fig. 3 denote the mean stretching of the fluorescently labeled $\mathrm{VWF}$ as obtained in a microfluidic chamber by video microscopy [10]. Note that the vWF is not attached or bound to a surface but freely suspended in the sheared solution. The shape of the experimental curve is similar to 


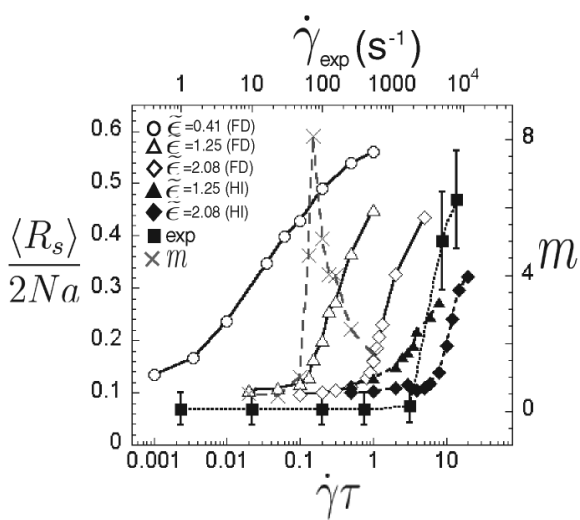

FIG. 3. Rescaled chain extension $\left\langle R_{s}\right\rangle / 2 N a$ for $N=50$ and different cohesive strengths $\tilde{\epsilon}$ with (HI, solid symbols) and without hydrodynamic effects (FD, open symbols) as a function of the rescaled shear rate $\dot{\gamma} \tau$. Crosses represent the rescaled variance $m$ of $R_{s}^{2}$ for $\tilde{\boldsymbol{\epsilon}}=1.25$ in the FD case. Experimental results (solid squares, upper horizontal axis) for $\mathrm{vWF}$ proteins were obtained in a microfluidic chamber [10].

simulation data for strongly collapsed polymers and, thus, confirms the presence of a well-defined unfolding transition.

We now introduce a simple scaling model for the globule unfolding in shear. We first note that, in the absence of thermal shape fluctuations, highly viscous spherical fluid drops are stable for any shear rate [11] (clearly, the viscosity of a polymeric globule is much higher than that of the surrounding solvent). This suggests a fluctuationinduced mechanism: Indeed, the numerically determined critical shear rate depends strongly on temperature (as shown below). The snapshots in Fig. 2 identify polymeric protrusions as the prevalent excitation mode. Approximating the globule shape as a sphere of radius $R$, the hydrodynamic drag force $f$ on a protruding polymer segment of length $l$ [see Fig. 4(a) for a schematic drawing] is in the freely draining (FD) case given by $f_{\mathrm{FD}} \sim \dot{\gamma} R l /\left(\mu_{0} a\right)$ [15]. In the hydrodynamic case, there is no slip at the globule-solvent boundary, but the difference between solvent and sphere velocity gradually increases away from the sphere surface. The force on a protrusion is obtained by integrating the hydrodynamic drag over the protrusion contour using the analytical expression for the flow profile around a sphere in shear flow [16]. The resulting radial force is maximal for an orientation $\theta=45^{\circ}$ and to leading order in $l / R$ reads $f_{\mathrm{HI}} \sim \dot{\gamma} l^{3} /\left(a \mu_{0} R\right)$, as will be explained in more depth in a longer paper [17]. The maximal tangential force is obtained for $\theta=0^{\circ}$ and scales identically. Not too close to the collapse point, the restoring force on a protrusion should be linear in $\tilde{\epsilon}$ and scale as $f_{\text {pro }} \sim$ $\left(k_{B} T / a\right) \Delta \tilde{\boldsymbol{\epsilon}}(l / a)^{\alpha-1}$, where the shift in the interaction parameter $\Delta \tilde{\boldsymbol{\epsilon}}=\left(\tilde{\boldsymbol{\epsilon}}-\tilde{\boldsymbol{\epsilon}}_{\mathrm{col}}\right)$ accounts for the configurational entropy loss in the globule. For a protrusion much longer than the globule/solvent interfacial width, one has $\alpha=1$, while for a shorter protrusion, one expects $\alpha=2$ in the harmonic approximation. From the equipartition theo- rem, the typical protrusion length $l$ follows as $l \sim$ $a \Delta \tilde{\boldsymbol{\epsilon}}^{-1 / \alpha}$. Comparing the hydrodynamic drag $f_{\mathrm{FD}}$ or $f_{\mathrm{HI}}$ (which tends to elongate protrusions) with the cohesive force $f_{\text {pro }}$ (which pulls protrusions back into the globule) and inserting the typical protrusion length, one obtains for the threshold shear rate $\dot{\gamma}^{*}$ :

$$
\dot{\gamma}^{*} \tau \sim \begin{cases}\Delta \tilde{\epsilon}^{2 / \alpha} a / R & (\mathrm{FD}), \\ \Delta \tilde{\epsilon}^{4 / \alpha} R / a & (\mathrm{HI}) .\end{cases}
$$

Note that the dependence on the globule radius is opposite when hydrodynamic effects are included. In the HI case, the critical shear rate increases as $R$ grows, since a larger sphere leads to a reduced fluid shear rate at its surface $[16,17]$. Our argument identifies the critical shear rate at which the hydrodynamic drag on a typical protrusion is large enough to overcome the restoring force from monomer-monomer attractions, similar to classical nucleation theory. Global free energy arguments are irrelevant, since a polymeric protrusion nucleus which has not reached the threshold length will be wrapped around the incessantly rotating globule and be eliminated within one period of revolution.

In Fig. 4(b), we compare simulation results for the critical shear $\dot{\gamma}^{*} \tau$ with the scaling prediction for the FD (using $\alpha=1$, solid line with slope 1) and HI cases ( $\alpha=2$, dashed line with slope -1 ), confirming the different scaling dependence on globule radius in the two cases. In the simulation, the globule radius $\tilde{R}_{0}$ is defined as the extension in the absence of shear, i.e., $\tilde{R}_{0}=\left\langle R_{s} / a\right\rangle_{\dot{\gamma}=0}$. Different values of $\alpha$ point to a sharp globule-solvent interface in the FD case (characterized by $\alpha=1$ ) and a presumably hydrodynamically roughened interface in the HI case $(\alpha=2)$. Deviations of the HI data from the scaling prediction [the dotted line in Fig. 4(b) has slope 0.69 and is a best fit to the data] are brought out more clearly in Fig. 4(c), where we plot the rescaled critical shear rate $\dot{\gamma}^{*} \tau \tilde{R}_{0}$ for the FD case and $\dot{\gamma}^{*} \tau / \tilde{R}_{0}$ for the HI case as a function of the cohesion variable $\Delta \tilde{\varepsilon}$. The freely draining data now exhibit two regimes: Close to the collapse transition, one finds $\alpha=2$ characteristic of protrusions shorter than the globule interfacial width, and further away the data are described well by $\alpha=1$, as expected; the crossover occurs at $\Delta \tilde{\boldsymbol{\epsilon}} \approx 0.4$. In the limited range available for the critical shear rate, the hydrodynamic data show behavior close to the scaling prediction; the broken line has slope 2 and corresponds to $\alpha=2$, and the dotted line has slope 1.38. Reasons for deviations are probably a breakdown of the simplified assumption about the spherical shape of the globule and the globule-solvent interface, which are both perturbed by hydrodynamic drag effects.

Our studies reveal the scaling of the critical shear rate for unfolding of globular polymers with various parameters. The particular value of $\dot{\gamma}^{*}$ depends strongly on the attraction forces between monomers and on the size of the polymer. Coming back to vWF in the bloodstream, with carefully tuned globule size and cohesive energy, unfold- 

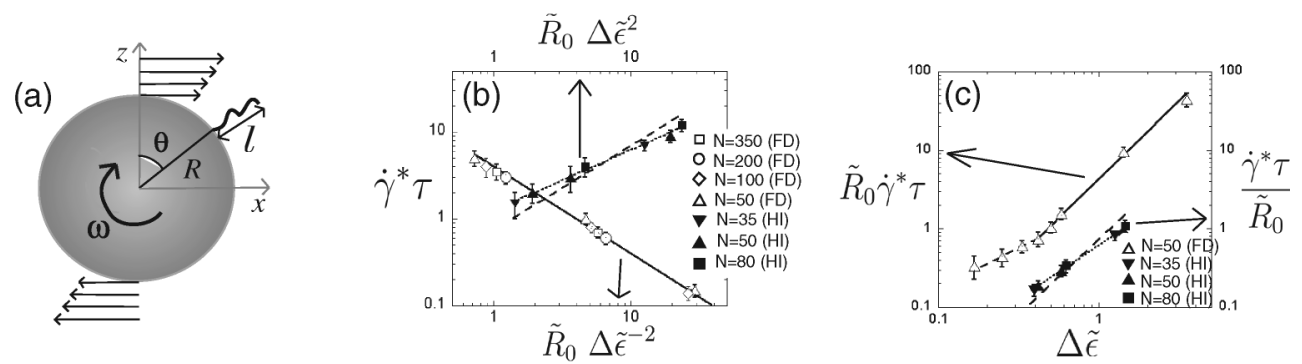

FIG. 4. (a) In the scaling model, a polymer strand of length $l$ protrudes from the spherical globule of radius $R$, which is rotating in the shear flow. (b) Dimensionless critical shear rate $\dot{\gamma}^{*} \tau$ for the FD and HI cases (open and solid symbols, respectively) as a function of the rescaled radius according to Eq. (2). (c) Rescaled critical shear rate $\tilde{R}_{0} \dot{\gamma}^{*} \tau$ as a function of $\Delta \tilde{\epsilon}$ for $N=50$ in the FD case (open triangles). The solid symbols correspond to the rescaled critical shear rate $\dot{\gamma}^{*} \tau / \tilde{R}_{0}$ in the HI case. In both graphs, the solid and dashed lines correspond to the scaling predictions with $\alpha=1$ and $\alpha=2$, respectively. The dotted lines are fits to the data; see text.

ing can occur preferentially near the surface of a capillary where the flow gradient and, thus, the shear rate are maximal and where presumably the unfolding of vWF has an important physiological function [8]. Converting Eq. (2) to physical variables, the critical shear in the experimentally relevant $\mathrm{HI}$ case scales as $\dot{\gamma}^{*} \sim \tau^{-1}(\Delta \tilde{\epsilon})^{4 / \alpha} \tilde{R}_{0} \sim$ $\eta^{-1} a^{-3}(\Delta \epsilon)^{4 / \alpha} N^{1 / 3}\left(k_{B} T\right)^{1-4 / \alpha}$ and, thus, shows a pronounced temperature dependence, where $\Delta \epsilon$ is a measure of the temperature-independent contact energy between two monomers. Let us discuss two limits for this contact energy: In the case of unspecific binding, one would expect the energy to scale with the contact area between monomers $\Delta \epsilon \sim a^{2} \sigma$, where $\sigma$ denotes the interaction energy per unit area, leading to $\dot{\gamma}^{*} \sim a^{8 / \alpha-3} \sigma^{4 / \alpha} N^{1 / 3}$. For any value $\alpha<8 / 3$, an increase in $a$ would increase $\dot{\gamma}^{*}$. In the case of specific monomer binding (probably more relevant for vWF), $\Delta \epsilon$ is independent of $a$, and thus $\dot{\gamma}^{*} \sim a^{-3} N^{1 / 3}$. The critical shear rate is seen to depend strongly and inversely on monomer size and only to a lesser degree on monomer number or, equivalently, polymer length. Let us now obtain numerical prefactors: A collapsed chain with $N=50$ and a monomer-monomer cohesive strength $\epsilon \sim$ $2 k_{B} T$ (which is a typical number for hydrogen bonds and probably of the right order for vWF, which unfolds already upon adsorption to a mildly hydrophobic surface [9]) unfolds at $\dot{\gamma}^{*} \tau \approx 9$ in the HI case (see Fig. 3). In order for such a protein to unfold at a typical shear rate in a human capillary of $\dot{\gamma} \sim 1000 \mathrm{~s}^{-1}$ [8] and using for the viscosity of water $\eta=10^{-3} \mathrm{~Pa}$, the required monomer radius $a$ turns out to be of the order of $a \sim 100 \mathrm{~nm}$, which is close to the actual size of a vWF monomer [9]. Our results thus suggest that the vWF monomer is so large in order for it to unfold at physiological shear rates.

In summary, we have shown that collapsed chains under shear unfold above a well-defined threshold shear rate. Hydrodynamic effects are crucial and change the dependence of the critical shear rate on the length of the polymer compared to the free draining case. Qualitative agreement with the unfolding of the vWF protein in a microfluidic chamber is obtained, and the importance of the monomer size is elucidated. The shear-induced unfolding of proteins and globular polymers is relevant for food-processing and medical or biotechnical applications.

Financial support by the Bavarian Elite Fund "Complex Interfaces," the Deutsche Forschungsgemeinschaft (SPP1164 and SFB486), the Center for Interdisciplinary Medical Research Münster, and the NSF is acknowledged.

[1] P. G. De Gennes, J. Chem. Phys. 60, 5030 (1974).

[2] D. E. Smith, H. P. Babcock, and S. Chu, Science 283, 1724 (1999).

[3] R. G. Larson J. Rheol. (N.Y.) 49, 1 (2005).

[4] M. Doi and S. F. Edwards, J. Chem. Soc., Faraday Trans. 75, 38 (1979).

[5] P. S. Doyle, B. Ladoux, and J.-L. Viovy, Phys. Rev. Lett. 84, 4769 (2000).

[6] A. Buguin and F. Brochard-Wyart, Macromolecules 29, 4937 (1996).

[7] J. E. Sadler, Annu. Rev. Biochem. 67, 395 (1998).

[8] M. H. Kroll et al., Blood 88, 1525 (1996).

[9] C. A. Siedlecki et al., Blood 88, 2939 (1996).

[10] S. W. Schneider, S. Nuschele, A. Wixforth, A. AlexanderKatz, R. R. Netz, and M. F. Schneider (to be published).

[11] H. A. Stone, Annu. Rev. Fluid Mech. 26, 65 (1994).

[12] R. M. Jendrejack, J.J. de Pablo, and M.D. Graham, J. Chem. Phys. 116, 7752 (2002).

[13] Y. Zhou, C. K. Hall, and M. Karplus, Phys. Rev. Lett. 77, 2822 (1996).

[14] W.W. Graessley, R. C. Hayward, and G. G. Grest, Macromolecules 32, 3510 (1999).

[15] Since simple shear consists of equal amounts of rotational and elongational flow, the sphere angular velocity is $\dot{\gamma} / 2$ for both the FD and HI scenarios [16]. For FD, the solvent/ sphere velocity difference at the surface is thus $\sim R \dot{\gamma}$, leading to a drag force of $\sim R \dot{\gamma} / \mu_{0}$ per monomer. Multiplication by the number of protruding monomers $\sim l / a$ yields $f_{\mathrm{FD}}$.

[16] T.G.M. van de Ven, Colloidal Hydrodynamics (Academic, London, 1989).

[17] A. Alexander-Katz and R. R. Netz (to be published). 